\title{
Experimental Quasi-Microwave Whole-Body Averaged SAR Estimation Method Using Cylindrical-External Field Scanning
}

\author{
Yoshifumi KAWAMURA $^{\dagger \text { a) }}$, Student Member, Takashi HIKAGE ${ }^{\dagger}$, Member, and Toshio NOJIMA ${ }^{\dagger}$, Fellow
}

\begin{abstract}
SUMMARY The aim of this study is to develop a new whole-body averaged specific absorption rate (SAR) estimation method based on the external-cylindrical field scanning technique. This technique is adopted with the goal of simplifying the dosimetry estimation of human phantoms that have different postures or sizes. An experimental scaled model system is constructed. In order to examine the validity of the proposed method for realistic human models, we discuss the pros and cons of measurements and numerical analyses based on the finite-difference time-domain (FDTD) method. We consider the anatomical European human phantoms and planewave in the $2 \mathrm{GHz}$ mobile phone frequency band. The measured wholebody averaged SAR results obtained by the proposed method are compared with the results of the FDTD analyses.

key words: whole-body averaged SAR, field measurement, scaled model, mobile phone frequency, plane-wave exposure system
\end{abstract}

\section{Introduction}

The whole-body averaged Specific Absorption Rate (WB$\mathrm{SAR}$ ) of $0.4 \mathrm{~W} / \mathrm{kg}$ is one of the vital physical quantities to specify the basic restrictions on human exposure to electromagnetic fields (EMF) regarding the adverse health effects prevention [1]. The reference levels as guidelines to control and estimate EMF exposure environments at frequencies above $10 \mathrm{MHz}$ are basically derived from the WBSAR associated with the plane-wave exposure condition [1]. Same process is applied in the telecommunications technology council report of MIC Japan [2] and the ANSI/IEEE C95.1-1999 standard [3].

The original relationships between the WB-SAR and the EMF reference levels were obtained through the theoretical analyses using the simple geometrical or block structure model with homogeneous tissue for human body [4]. However, since these relationships for practical human exposure situations might be complicated, more accurate and precise estimations based upon realistic shaped and anatomical human models are expected. Recently, high resolution numerical simulations have become possible through the use of advanced high-speed computers and 3-D EMF analysis software tools or original program codes [5]-[9]. Thus, advancements in the side of theoretical estimations are being achieved steadily. On the other hand, some experiments to investigate the same subject have been reported [9], [10]. The WB-SAR is estimated from the absorption power of

\footnotetext{
Manuscript received November 1, 2009.

$\dagger$ The authors are with the Graduate School of Information Science and Technology, Hokkaido University, Sapporo-shi, 0600814 Japan.

a)E-mail: kawamura@wtemc.ist.hokudai.ac.jp

DOI: 10.1587/transcom.E93.B.1826
}

the human body phantom model which is exposed to the plane-wave of known EMF strength in the anechoic chamber. For the absorption power measurements, 3-D scanning techniques using electric probes inserted into the phantom [10] or thermographic techniques [11] have been applied. One of the scanning techniques is widely used as an IEC standard system for the SAR measurement on hand-held devices. However, at microwave frequencies these techniques have a substantial problem that it is difficult to scan precisely all over the entire phantom body required for the WB-SAR measurements.

Our solution is to propose a novel WB-SAR estimation method based on the external cylindrical field scanning technique [12]. This method measures electric field strength by scanning a cylindrical surface of the closed region containing the phantom and the plane-wave radiation source inside. The similar scanning techniques have been employed to obtain the antenna radiation patterns [13], [14]. A key characteristic of the proposed method is that it can be easily applied to human phantoms that have different postures or sizes, because it is not necessary to obtain the electromagnetic components distributed inside of the human phantom.

This paper describes the experimental set-up for WBSAR estimations of scaled homogeneous human phantoms that have $2 / 3$ muscle-equivalent complex permittivity material and same standing posture. Whole-body absorption power is measured at $4 \mathrm{GHz}$ using a male adult or a child phantom. In addition, in order to confirm the applicability of the proposed method, numerical estimations of inhomogeneous human phantoms exposed to an ideal planewave are performed with the FDTD (Finite-difference Timedomain) method. The human phantom parameters are designed based upon Virtual Family Product series [15].

\section{Methodology}

\subsection{Whole-Body Averaged SAR Estimation Method}

It is a technique that assesses WB-SAR by measuring the radiation power on closed surface including human phantom and some radiation source. This method is based on scanning the EMF data in the near-field of the antenna when the radiation characteristic of the antenna is measured [13]. The principle of radiation energy balance of the system is shown in Fig. 1. In the figure, the absorption power $\left(W_{a b}\right)$ of the lossy material (phantom) is given by the following expression from the law of the conservation of energy: 


$$
W_{a b}=W_{\text {in }}-W_{\text {out }}
$$

where, $W_{\text {in }}$ (in watt) is a antenna output power and $W_{\text {out }}$ (in watt) is a total radiation power from the closed region. The power $W_{\text {out }}$ from the closed region is yielded as a surface integral of the Poynting vector $\mathbf{P}$ (in watts per square meter) over a closed surface $S$ :

$$
W_{\text {out }}=\iint_{S} \mathbf{P} \cdot \mathbf{n} d S
$$

where,

$$
\mathbf{P}=\frac{1}{2} \mathbf{E} \times \mathbf{H}^{*}
$$

Moreover, the WB-SAR can be derived from the absorption power $\left(W_{a b}\right)$ and weight of the lossy material as follows:

$$
\text { Whole-body averaged SAR }=\frac{W_{a b}}{\text { Weight }}
$$

\subsection{Field Scanning Method}

Three major approaches have been advanced for scanning EMFs. The types of scanning surfaces used are planar, cylindrical, and spherical. The advantages and disadvantages of using the different surfaces have been previously discussed [13]. The technique that used most often is spherical scanning. Spherical scanning makes highly accurate measurements possible since it can obtain the EMFs that are measured at the same length from the center position of the scanning surface. However, it is not suitable for an experimental system targeting the WB-SAR since this system requires an increase in the estimation space and for the measured object to be suspended in midair.

Cylindrical scanning and planar scanning are, however, suitable for practical experimental systems. This paper examines the application of cylindrical scanning.

The cylindrical scanning system has two dimensions: height interval $\Delta z$ and circumferential interval $\Delta \phi$. For these

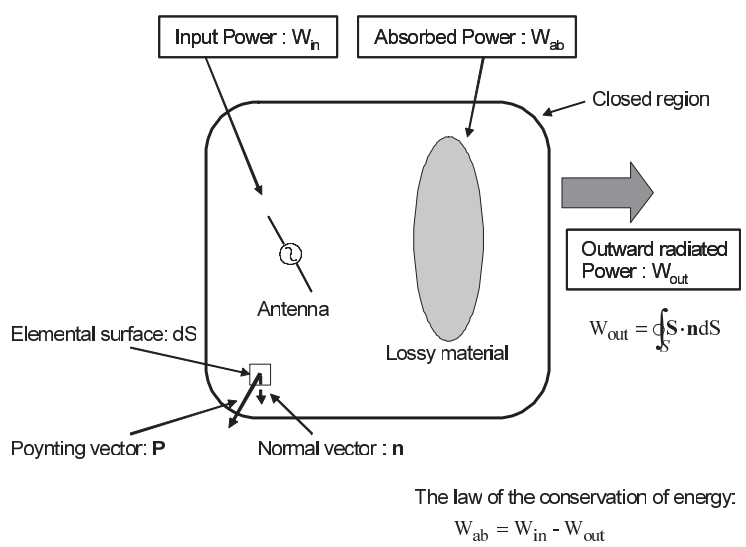

Fig. 1 The principle of radiation energy balance of the system based on the law of the conservation of energy. sampling intervals, the following expressions are applied [16].

$$
\Delta z \leq \frac{\lambda}{2} \frac{a}{\rho}, \quad \Delta \phi \leq \frac{\pi}{k \rho}=\frac{\lambda}{2 \rho}
$$

Thus, $k$ (in per meter) is shown as a wave number, $\lambda$ (in meter) is shown as wavelength, $\rho$ (in meter) is the radius of the minimum sphere surrounding the measured object and $a$ (in meter) is the radius of the cylindrical scanning surface. In this report, the sampling intervals are decided referring to the value derived from these conditions.

In order to include areas not usually considered, such as the upper and lower parts of the cylinder, the absorption power is subtracted from the radiation power when the lossy material is in the scanning surface as well as when the material is not in the scanning surface. Equation (2) is redefined (for cylindrical scanning) as follows:

$$
W_{\text {out }}=\iint_{S} \mathbf{P} \cdot \mathbf{n} d S=\sum_{i=0}^{N} \sum_{j=0}^{M} P_{r} \Delta z_{i}\left(\frac{a \Delta \phi_{j}}{\pi}\right)
$$

\section{Validation of Proposed Method Using Numerical Analysis}

\subsection{Numerical Model}

The numerical human model is shown in Fig. 2. This model is an enhanced high-resolution magnetic resonance imaging whole-body human model and was made as one of the Virtual Family Product series [15]. This represents a 34-yearold male adult. It consists of 77 inhomogeneous tissues; its height is about $1.74 \mathrm{~m}$ and weight is $70 \mathrm{~kg}$. The resolution of the model used is $2.0 \mathrm{~mm}$.

Numerical EMF analyses were performed using the FDTD method [17] since this method well suits the computation of large scale and high precision inhomogeneous programs. The FDTD numerical analysis was performed by commercial software SEMCAD X [18] which incorporates a number of advanced tools for the visualization and treatment of simulation results.

The numerical model of the SAR experiment evaluation system including the numerical human model is shown

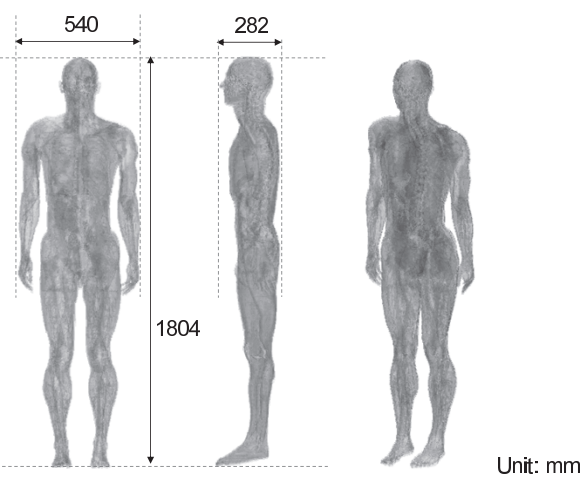

Fig. 2 Male adult model of Virtual Family Product. 


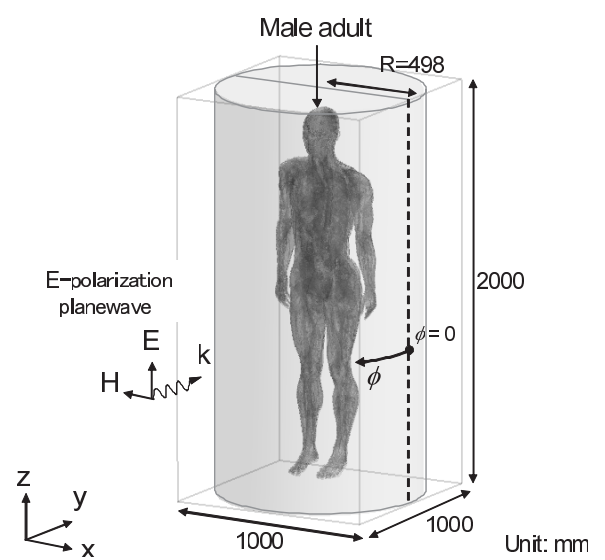

Fig. 3 Numerical analysis model.

Table 1 Computation parameters.

\begin{tabular}{|l|c|}
\hline Frequency & $2100 \mathrm{MHz}$ \\
\hline Resolusion & $2 \mathrm{~mm}($ cubic $)$ \\
\hline Scattering zone & $10 \mathrm{~mm}(5$ cells $)$ \\
\hline Absorbing B.C. & U-PML $(10$ Layer $)$ \\
\hline Total program size & $279 \mathrm{Mcells}$ \\
\hline Incident wave & E-polarized plane-wave \\
& $\left(1.0 \mathrm{~mW} / \mathrm{cm}^{2}\right)$ \\
\hline Material & Homogeneous \\
& $\left(\varepsilon_{r} 39.82, \sigma 1.49 \mathrm{~S} / \mathrm{m}\right.$, \\
& $\left.\rho 1000 \mathrm{~kg} / \mathrm{m}^{3}\right)$ \\
\cline { 2 - 2 } & Inhomogeneous \\
\hline
\end{tabular}

in Fig. 3. This calculation model assumes a plane-wave generated by a $2.1 \mathrm{GHz}$ cellular phone impacting the human body. The male adult is located at the center of the planewave irradiation area.

Table 1 describes the parameters of FDTD calculation and the dielectric properties. The cell size of the analysis model is $2.0 \mathrm{~mm}$. The computational space is $1000 \times 1000 \times$ $2000 \mathrm{~mm}^{3}(500 \times 500 \times 1000$ cells $)$. The absorbing boundary condition is a uniaxial perfectly matched layer (UPML) having 10 layers. The human model is composed of homogeneous tissue of $2 / 3$ muscle-equivalent or inhomogeneous tissues [19]. 2/3 muscle-equivalent tissue means that its conductivity and permittivity are $2 / 3$ times of the muscle's ones.

The frequency of the plane-wave is $2.1 \mathrm{GHz}$ and the plane-wave has uniform E polarization. The radiation power given as a power density is $1 \mathrm{~mW} / \mathrm{cm}^{2}$ based on ICNIRP public exposure reference levels [1] from the front side of the human body.

\subsection{Estimation Condition of Proposed Method}

The radiation power in a closed region was calculated using the EMF distributions given by the numerical analysis. In numerical analysis, an ideal probe that does not disturb the EMF distribution is assumed. Since the plane-wave source is located outside of the closed surface, the antenna input

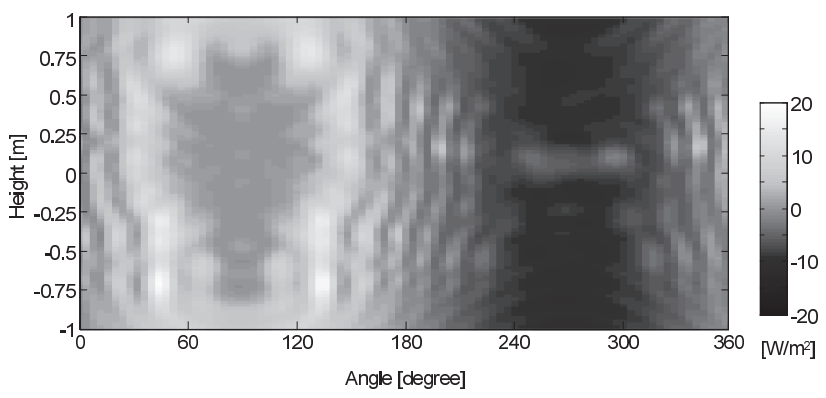

Fig. 4 Poynting vector (component of $P_{r}$ ) distribution on the cylindrical surface for homogeneous male adult phantom.

Table 2 Comparison of proposed method and IEEE C95.3 technique results.

\begin{tabular}{|c|c|c|c|c|}
\hline \multirow{2}{*}{} & \multicolumn{2}{|c|}{ Homogeneous } & \multicolumn{2}{c|}{ Inhomogeneous } \\
\cline { 2 - 5 } & $\begin{array}{c}\text { Proposed } \\
\text { method }\end{array}$ & $\begin{array}{c}\text { IEEE } \\
\text { technique }\end{array}$ & $\begin{array}{c}\text { Proposed } \\
\text { method }\end{array}$ & $\begin{array}{c}\text { IEEE } \\
\text { technique }\end{array}$ \\
\hline$W_{a b}[\mathrm{~W}]$ & 4.026 & 3.539 & 4.856 & 4.322 \\
\hline $\begin{array}{c}\text { WB-SAR } \\
{[\mathrm{W} / \mathrm{kg}]}\end{array}$ & 0.0587 & 0.0516 & 0.0672 & 0.0598 \\
\hline Variation & $+13.7 \%$ & - & $+12.4 \%$ & - \\
\hline
\end{tabular}

power $\left(W_{i n}\right)$ is $0 \mathrm{~W}$. The absorbed power $\left(W_{a b}\right)$ equals to the radiation power $\left(W_{\text {out }}\right)$ from the closed region. The FDTD analysis results are initially given in Cartesian coordinates. Accordingly, those results are converted into Cylindrical Polar Coordinates and the Poynting vector distributions on the cylindrical surface are obtained.

The sampling intervals on the cylindrical surface are derived from the sampling assumption of expression (5). The height interval is $20 \mathrm{~mm}$ and the circumferential interval is 4 degrees. Here, the sampling intervals are $20 \mathrm{~mm} \times 20 \mathrm{~mm}$.

\subsection{Result}

The Poynting vector distribution obtained when using homogeneous male adult phantom is shown in Fig. 4. The horizontal axis is angle $\phi$ and the vertical axis is height $z$ of the cylindrical scanning surface. The plane-wave comes from 270 degrees direction perpendicular to the closed surface. In this numerical model, the Poynting vectors take negative values on the surface where the plane-wave enters from outside of the closed region.

The absorbed power and WB-SAR estimation results derived from the Poynting vector distribution for the male adult phantom are shown in Table 2. This table includes the WB-SAR estimation result using FDTD dosimetric calculation based on IEEE Standard C95.3 [20]. In this standard technique, the WB-SAR is evaluated by averaging the SAR distributions inside of the phantom. The human phantom weight is $68.55 \mathrm{~kg}$ (homogeneous tissue) and $72.24 \mathrm{~kg}$ (inhomogeneous tissues) as calculated from FDTD cells of the 
numerical analysis model. The WB-SAR of the homogeneous phantom is $0.0587 \mathrm{~W} / \mathrm{kg}$ using the proposed method and $0.0516 \mathrm{~W} / \mathrm{kg}$ by the IEEE calculation technique. In addition, the WB-SAR of the inhomogeneous phantom is $0.0672 \mathrm{~W} / \mathrm{kg}$ using the proposed method and $0.0598 \mathrm{~W} / \mathrm{kg}$ by the IEEE calculation technique. The WB-SAR using the proposed method is within $13 \%$ of the IEEE calculation results. This confirms that the proposed method is suitable for both homogeneous and inhomogeneous phantoms.

As mentioned in Sect.2.2, it is difficult for the WBSAR estimation method using cylindrical field scanning method to experimentally estimate the Poynting vectors on the top and bottom surfaces. However, the numerical estimation result of the absorbed power including the top and bottom areas using cylindrical scanning is $3.813 \mathrm{~W}$ for the homogeneous phantom. This means that the error attributed to this incompleteness is less than $8 \%$. Thus, the validity of the field scanning proposal has been confirmed.

\section{Measurement Condition and System}

The experimental set-up based on the proposed method is described. The far-field relationship between $\mathrm{E}$ and $\mathrm{H}$ is assumed. This makes it possible to conduct power estimations using the wave impedance of free space $(377 \Omega)$ and the electric field distributions. When the radiation source is located inside the closed region, some amount of the radiation power leaks from the top and bottom surfaces. Assuming the leak power difference is negligible between the situations with and without the phantom, the antenna input power should be corrected by the leak power. Accordingly, the leak power without the phantom is measured in advance.

\subsection{Experimental Set-Up}

A block diagram of the proposed experimental set-up for WB-SAR estimation is shown in Fig. 5. The experimental set-up consists of the radiation system, the electric field measurement system, and the physical phantom whose dielectric properties match those of the human body.

This set-up assumes a human exposed to a $2 \mathrm{GHz}$ Epolarized plane-wave. The power density of the plane-wave is $1 \mathrm{~mW} / \mathrm{cm}^{2}$. To estimate plane-wave exposure at mobile radio frequencies on the human body, the direct approach is to use a full-size model. Instead, this study uses a scaled model to minimize the estimation space requirements and so

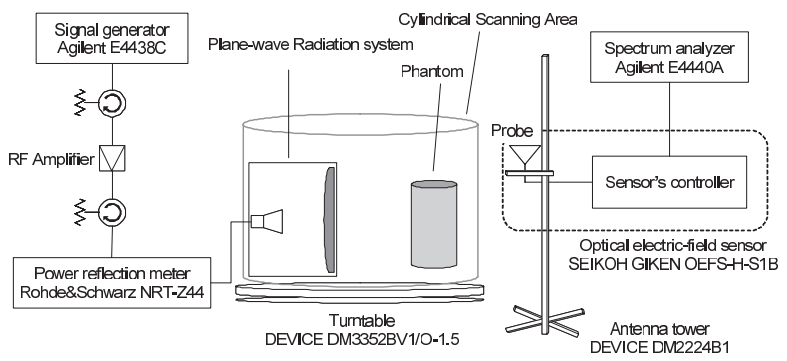

Fig. 5 Block diagram of the proposed experimental set-up for WB-SAR estimation. permit the use of practical anechoic chambers. In an evaluation that includes lossy materials such as the human phantom, both the electric constant and the scale of the lossy material should change. In this study, a 1/2 scale model is used. The measurement systems are automatically controlled by program code through GPIB.

\subsection{Scaled Modeling}

In order to subject the human body to uniform plane-wave excitation in the cellular phone frequency band $(2.0 \mathrm{GHz})$, a large-scale evaluation area (of the order of 10's of meters) is required.

Accordingly, the evaluation area was reduced by scaling both antenna size and frequency. In an evaluation system including lossy materials such as human bodies, both the dielectric constant and the scale of the lossy material should change appropriately. The reduced scale relation between the full-scale model and a model with a scale factor of $k$ [21], [22] is shown in Table 3. For a scaled model with a scale factor of $k$, the dielectric constants of the scaled model are the equal relative permittivity and the $1 / k$ times conductivity.

In this paper, a $1 / 2$ scale model is used. Since a $1 / 2$ scale factor is used, object sizes are reduced by a half and the antenna radiation frequency is $4.0 \mathrm{GHz}$.

\subsection{Radiation System}

The radiation source outputs a $4 \mathrm{GHz}$ continuous wave. A plane-wave radiation system that generates a uniform planewave is required, since the guideline of the EMF strength assumes an ideal plane-wave that vertically enters the human body.

In order to easily generate a plane-wave with sharp directivity, horn antennas with sharp directivity are used as radiators. Figure 6 illustrates the plane-wave radiation system that was used. Only a localized surface of the human phantom could be exposed without any measures since the electric field strength in front of the horn antenna is comparatively high. Thus a dielectric lens was designed and set in front of the horn antenna. The relative permittivity of the dielectric lens is 3.7. The dielectric lens aligns the amplitude and phase of the wave from the horn antenna.

We used a double ridge horn antenna in order to realize wideband characteristic. The nominal frequency range of the antenna is from $1 \mathrm{GHz}$ to $5 \mathrm{GHz}$. The overall size of the radiation system is $800 \times 750 \times 1300 \mathrm{~mm}^{3}$. The radiation system irradiates a plane-wave whose excitation area is

Table 3 Electrical properties for full scale model and scale model.

\begin{tabular}{|l|c|c|}
\hline Paraneter & Full scale model & Scale model \\
\hline Length & $l$ & $l^{\prime}=k l$ \\
\hline Frequency & $f$ & $f^{\prime}=(1 / k) f$ \\
\hline Permittivity & $\epsilon$ & $\epsilon^{\prime}=\epsilon$ \\
\hline Conductivity & $\sigma$ & $\sigma^{\prime}=(1 / k) \sigma$ \\
\hline
\end{tabular}




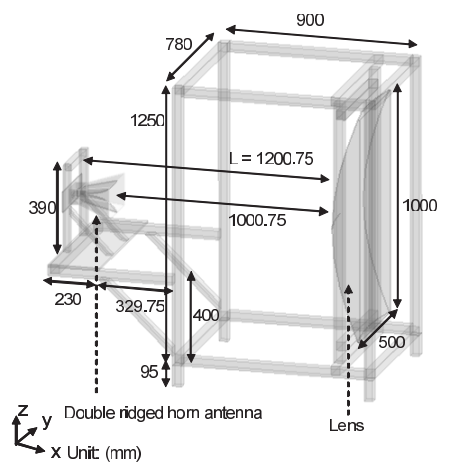

(a) Numerical model

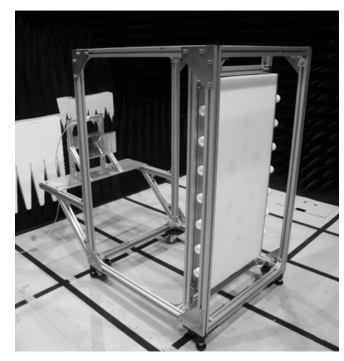

(b) Experimental radiation system
Fig. 6 Experimental plane-wave exposure system.

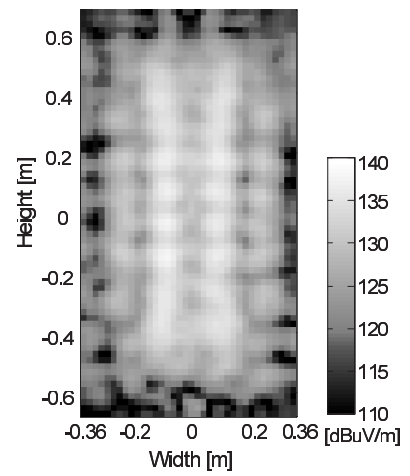

(a) $E_{z}$

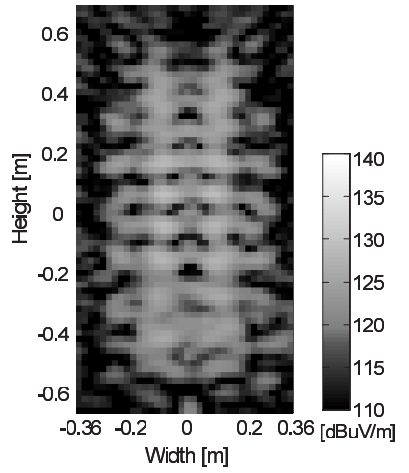

(b) $\mathrm{E}_{\mathrm{y}}$

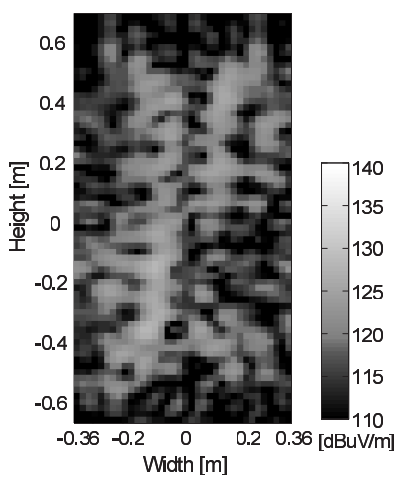

(c) $E_{x}$

Fig. 7 Measured electric field distributions on the planar boundary at the position of $500 \mathrm{~mm}$ from the dielectric lens.

$1000 \times 500 \mathrm{~mm}^{2}$.

The measured electric-field components on the planer surface at the position of $500 \mathrm{~mm}$ from the dielectric lens are shown in Figs. 7(a)-(c). The main polarized component of the radiation system is vertical polarization $(\mathrm{Ez})$. The antenna input power is $23 \mathrm{dBm}$. The Ez strength of the areairradiated plane-wave is within $3 \mathrm{~dB}$. The field strength of cross-polarized components decreases by $10 \mathrm{~dB}$ compared to the field strength of the main polarized component.

The averaged electric power density in the plane-wave excitation area is derived. The electric power density is assumed to be a far field area in this space. The averaged

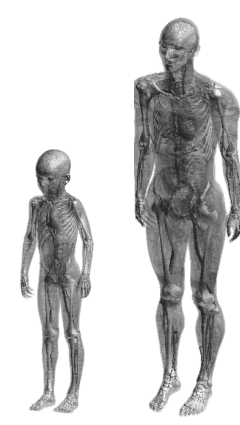

(a) Numerical Model

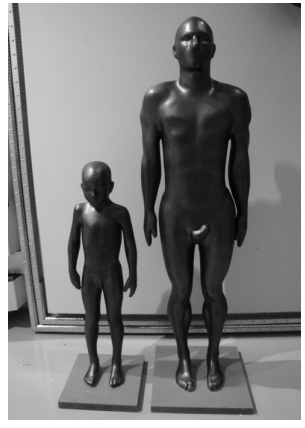

(b) Solid Phantom
Fig. 8 Standing human phantom based on numerical CAD data (Left phantom is male child and right phantom is male adult based on Virtual Family Product series).

Table 4 Scaled solid phantom parameters.

\begin{tabular}{|c|c|c|}
\hline & Male Adult & Male Child \\
\hline Size and Weight & $\begin{array}{l}\text { height: } 90 \mathrm{~cm} \\
\text { weight: } 8.54 \mathrm{~kg}\end{array}$ & $\begin{array}{l}\text { height: } 59 \mathrm{~cm} \\
\text { weight: } 2.28 \mathrm{~kg}\end{array}$ \\
\hline Material & \multicolumn{2}{|c|}{$\begin{array}{c}\text { 2/3 muscle-equivalent tissue } \\
\text { (homogeneous) }\end{array}$} \\
\hline Tissue parameters & \multicolumn{2}{|c|}{$\epsilon_{r}: 36.0, \sigma: 2.1 \mathrm{~S} / \mathrm{m}$} \\
\hline
\end{tabular}

electric power density in the plane-wave excitation area is $0.154 \mathrm{~W} / \mathrm{m}^{2}$. Hence, the electric power density is $1 / 65$ times the reference level of ICNIRP $\left(1 \mathrm{~mW} / \mathrm{cm}^{2}\right.$ at $\left.2 \mathrm{GHz}\right)$.

\subsection{Field Sensor and Positioner}

The radiation power from the cylindrical closed surface is obtained by electric field distribution measurement using a 3-axis optical electric-field sensor (SEIKOH GIKEN OEFSH-S1B). The optical sensor has uncertainty values in the range of $\pm 0.5 \mathrm{~dB}$. The translation error of Poynting vector is ignored. The cylindrical scanning system is composed of the antenna tower (DEVICE DM2224B1) and the turntable (DEVICE DM3352BV1/O-1.5). The minimum steps of the antenna tower and the turntable are $10 \mathrm{~mm}$ and 0.1 degrees, respectively. The sampling intervals in cylindrical scanning are $20 \mathrm{~mm}$ in height and 1 degree in circumference. The human phantom is separated by $0.5 \mathrm{~m}$ from the front surface of the lens.

The optical sensor is mounted on the antenna tower. The optical sensor output is connected to the sensor controller through $20 \mathrm{~m}$ of optical fiber cable. The controller output is fed to the input of spectrum analyzer (Agilent E4440A).

\subsection{Solid Phantom Made from Silicone Rubber}

Two physical human phantoms were made based on enhanced high-resolution European human CAD data [15]. A 34-year-old male adult and a 6-year old male child model were used, as shown in Fig. 8. The full-size male child has 
about $1.17 \mathrm{~m}$ in height and $19.5 \mathrm{~kg}$ in weight. The phantom parameters are summarized in Table 4. The human phantom is composed of $2 / 3$ muscle-equivalent tissue [19]. The phantoms used in this study consist of carbon nanotubes embedded in silicone rubber [23]. This material has a specific gravity below $1.3 \mathrm{~g} / \mathrm{cm}^{3}$. The measured complex dielectric constants of the male adult phantom is 31.3 in relative permittivity and $1.93 \mathrm{~S} / \mathrm{m}$ in conductivity. The variations of the complex dielectric constants are within $20 \%$.

\section{Result}

\subsection{Validity of Experimental Set-Up Using Simplified} Rectangular Phantom

Basic geometrical phantoms must be examined when confirming the experimental set-up. Such phantoms are a sphere, rectangular or spheroid, and so on. As for the basic geometrical structure, a theoretical analysis based on electromagnetic theory has already been done [24], [25].

In this paper, a rectangular phantom is used. The phantom model offers highly accurate WB-SAR estimations, because this phantom has a simplified structure that is affected by the staircase approximation employed in the FDTD method.

The square planar shape phantom used for the numerical calculations and the measurements is shown in Fig. 9. It has dimensions of $400 \mathrm{~mm} \times 400 \mathrm{~mm} \times 50 \mathrm{~mm}$. Its weight is $8.0 \mathrm{~kg}$ based on the fact that mass density of the phantom is regarded as $1000 \mathrm{~kg} / \mathrm{m}^{3}$. The target values of relative permittivity and conductivity for the rectangular phantom are 45.0 and $8.0 \mathrm{~S} / \mathrm{m}$, respectively.

The WB-SAR estimation result using the proposed method is $0.111 \mathrm{~W} / \mathrm{kg}$. The WB-SAR result using FDTD analysis with ideal plane-wave is $0.093 \mathrm{~W} / \mathrm{kg}$. These values are normalized to the reference level of the ICNIRP Guideline. The difference of these results is within $20 \%$.

\subsection{WB-SAR Estimation Result}

The experimental set-up for the male adult human phantom in the anechoic chamber is shown in Fig. 10. The electric power of the plane-wave radiation system was $23 \mathrm{dBm}$. Thus, the averaged electric power density of the plane-wave excitation space of the radiation system is $0.154 \mathrm{~W} / \mathrm{m}^{2}$. The electric field distributions on the cylindrical scanning area

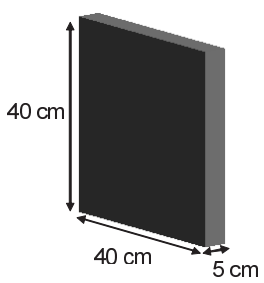

(a) Numerical Model

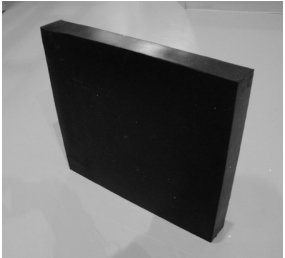

(b) Solid Phantom
Fig. 9 Simplified rectangular phantom. without and with the male adult phantom are shown in Fig. 11 and Fig. 12, respectively. However, the component of Er not used in the power evaluation is omitted. The evaluation cylinder has a height of $1.8 \mathrm{~m}$ and diameter of $3.0 \mathrm{~m}$.

Next, the Poynting vector on cylindrical surface is derived based on the electric field data. The measured absorption power and WB-SAR estimation results for the male adult and male child models are summarized in Table 5 . The WB-SARs are calculated using the weight of the numerical human phantom models. The absorbed power is normalized for the power density of exposed plane-wave of $1 \mathrm{~mW} / \mathrm{cm}^{2}$.

The measured WB-SAR for the male adult is

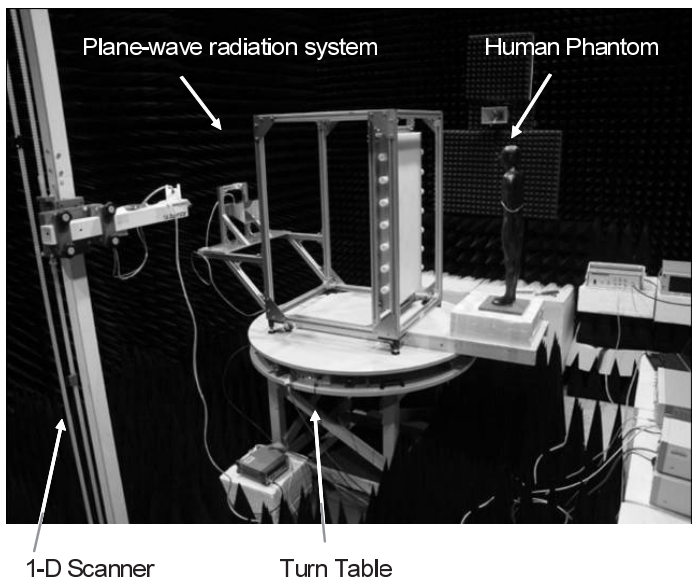

Fig. 10 Proposed experimental set-up in anechoic chamber.

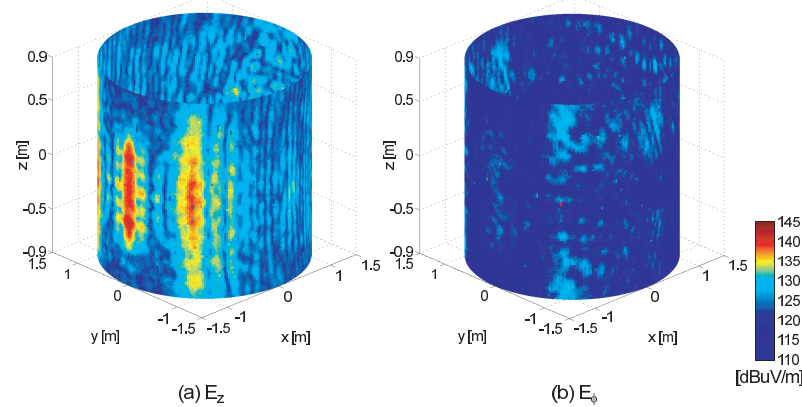

Fig. 11 Measured electric field distributions without the lossy material on cylindrical scanning area.

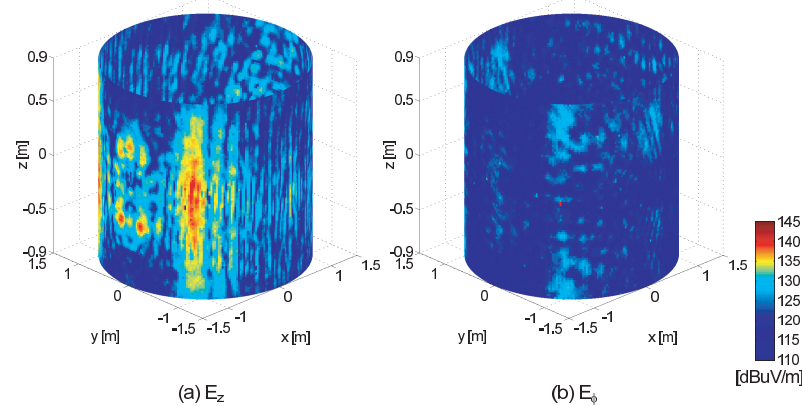

Fig. 12 Measured electric field distributions with the male adult phantom on cylindrical scanning area. 
Table 5 Comparison of measurement results based on proposed method and IEEE C95.3 calculation results.

\begin{tabular}{|c|c|c|c|c|}
\hline \multirow{2}{*}{} & \multicolumn{2}{|c|}{ Male adult } & \multicolumn{2}{c|}{ Male child } \\
\cline { 2 - 5 } & $\begin{array}{c}\text { Meas. } \\
\text { Result }\end{array}$ & $\begin{array}{c}\text { Calc. } \\
\text { Result }\end{array}$ & $\begin{array}{c}\text { Meas. } \\
\text { Result }\end{array}$ & $\begin{array}{c}\text { Calc. } \\
\text { Result }\end{array}$ \\
\hline $\begin{array}{c}\text { Normalized } \\
W_{a b}[\mathrm{~W}]\end{array}$ & 4.825 & 3.873 & 2.406 & 1.824 \\
\hline $\begin{array}{c}\text { WB-SAR } \\
{[\mathrm{W} / \mathrm{kg}]}\end{array}$ & 0.0704 & 0.0565 & 0.131 & 0.100 \\
\hline
\end{tabular}

$0.0704 \mathrm{~W} / \mathrm{kg}$. A numerical estimated WB-SAR for the male adult model exposed to ideal plane-wave is $0.0565 \mathrm{~W} / \mathrm{kg}$ obtained by the IEEE standard calculation method. In addition, the measured and calculation result for the male child is $0.131 \mathrm{~W} / \mathrm{kg}$ and $0.100 \mathrm{~W} / \mathrm{kg}$, respectively. The measured WB-SAR results are $30 \%$ larger than calculated results for both the adult and child phantom cases. Improvement of these uncertainties is one of the future study issues.

\section{Conclusions}

This paper introduces an actual experimental set-up for WBSAR measurements based on the cylindrical field scanning method. Several measurement results of WB-SAR exposure were reported. The experimental results agree with the numerical ones in terms of a simplified rectangular phantom and a few human phantoms. This technique supports changes in phantom model.

Future works include precise numerical estimations including the phantom and the radiation source that are identical condition to the experimental system, and investigation of experimental characteristics on translation error of Poynting vector. Additionally, the experimental WB-SAR estimation of human phantoms that have different postures or sizes will be conducted.

\section{Acknowledgments}

This work is supported by a Grant-in-Aid from the Ministry of Internal Affairs and Communications (MIC) of Japan.

\section{References}

[1] ICNIRP Guidelines, "Guidelines for limiting exposure to timevarying electric, magnetic, and electromagnetic fields (up to 300 GHz)," Health Phys., vol.74, no.4, pp.494-522, 1998.

[2] "Measurement of SAR from mobile phone terminals and other terminals that are intended for use in close proximity to the side of the head," Telecommunications Technology Council Report, Deliberation no.118, 2000

[3] ANSI/IEEE C95.1-1999, IEEE Standard for Safety Levels with Respect to Human Exposure Toradio Frequency Electromagnetic Fields, $3 \mathrm{kHz}$ to $300 \mathrm{GHz}$.

[4] S.M. Michaelson and J.C. Lin, Biological Effects and Health Implications of Radiofrequency Radiation, Plenum Press, New York and London, 1987.

[5] P.J. Dimbylow, "Fine resolution calculations of SAR in the human body for frequencies up to $3 \mathrm{GHz}$," Phys. Med. Biol., vol.47, pp.2835-2846, 2002.
[6] E. Conil, A. Hadjem, F. Lacroux, M.F. Wong, and J. Wiart, "Variability analysis of SAR from $20 \mathrm{MHz}$ to $2.4 \mathrm{GHz}$ for different adult and child models using finite-difference time-domain," Phys. Med. Biol., vol.53, pp.1511-1525, 2008

[7] J. Wang, O. Fujiwara, S. Kodera, and S. Watanabe, "FDTD calculation of whole-body average SAR in adult and child models for frequencies from $30 \mathrm{MHz}$ to $3 \mathrm{GHz}$," Phys. Med. Biol., vol.51, pp.4119-4127, 2006.

[8] M.-C. Gosselin, A. Christ, S. Kuhn, and N. Kuster, "Dependence of the occupational exposure to mobile phone base stations on the properties of the antenna and the human body," IEEE Trans. Electromagn. Compat., vol.51, no.2, pp.227-235, May 2009.

[9] R.P. Findlay, A.-K. Lee, and P.J. Dimbylow, "FDTD calculations of SAR for child voxel models in different postures between $10 \mathrm{MHz}$ and $3 \mathrm{GHz}$," Radiat. Prot. Dosimetry, pp.1-6, 2009.

[10] A.W. Guy, C.-K. Chou, and B. Neuhaus, "Average SAR and SAR distributions in man exposured to $450-\mathrm{MHz}$ radiofrequency radiation," IEEE Trans. Microw. Theory Tech., vol.MTT-32, no.8, pp.452-762, 1984

[11] Y. Okano, K. Ito, and H. Kawai, "Solid phantom composed of glycerin and its application to SAR estimation," IEICE Trans. Commun. (Japanese Edition), vol.J83-B, no.4, pp.534-543, April 2000.

[12] Y. Kawamura, T. Hikage, and T. Nojima, "Experimental wholebody average SAR estimation system using cylindrical field scanning method," 2009 International Symposium on Electromagnetic Compatibility, 21S4-4, pp.257-260, Kyoto, Japan, July 2009.

[13] G.E. Evans, Antenna Measurement Techniques, Artech House Publishers, 1990.

[14] J. A-Hansen, "On cylindrical near-field scanning techniques," IEEE Trans. Antennas Propag., vol.AP-28, no.2, pp.231-234, March 1980

[15] A. Christ, W. Kainz, E. Hahn, K. Honegger, J. Shen, W. Rascher, R. Janka, W. Bautz, B. Kiefer, P. Schmitt, H. Hollenbach, J. Chen, A. Kam, E. Neufeld, M. Oberle, and N. Kuster, "The virtual family project - Development of anatomical whole-body models of two adults and two children," Proc. 23rd Annual Review of Progress in Applied Computational Electromagnetics (ACES) 2007, 2007.

[16] T.B. Hansen, "Probe-corrected near-field measurements on a truncated cylinder," J. Acoust. Soc. Am., vol.119, no.2, pp.792-807, Feb. 2006.

[17] A. Taflove, Computational Electrodynamics (The Finite-Difference Time-Domain Method), Artech House, Boston, 1995.

[18] Schmid \& Partner Engineering AG, SEMCAD-X (http://www. semcad.com)

[19] C. Gabriel, "Compilation of the dielectric properties of body tissues at RF and microwave frequencies," Brooks Air Force Technical Report AL/OE-TR-1996-0037, 1996.

[20] IEEE C95.3-2002, Recommended Practice for Measurements and Computations of Radio Frequency, Electromagnetic Fields With Respect to Human Exposure to Such Fields, $100 \mathrm{kHz}-300 \mathrm{GHz}$

[21] A. Miyata, Y. Yokota, K. Fujimoto, and T. Maeda, "A proposal of UWB scale-up model phantoms for the scale model evaluations of UWB antennas including the effects of human body," IEICE Trans. Commun. (Japanese Edition), vol.J91-B, no.7, pp.801-805, July 2008

[22] C.A. Balanis, Antenna Theory: Analysis and Design, 3rd ed., pp.1044-1045, Wiley-Interscience, 2005.

[23] T. Hikage, Y. Sakaguchi, T. Nojima, and Y. Koyamashita, "Development of lightweight solid phantom composed of silicone rubber and carbon nanotubes," Proc. 2007 IEEE EMC Symposium, TH-AM-34, USA, 2007.

[24] J.A. Stratton, Electromagnetic Theory, McGraw-Hill, 1941.

[25] H.N. Kritikos and H.P. Schwan, "The distribution of heating potential inside lossy spheres," IEEE Trans. Biomed. Eng., vol.BME-22, no.6, pp.457-462, 1975. 


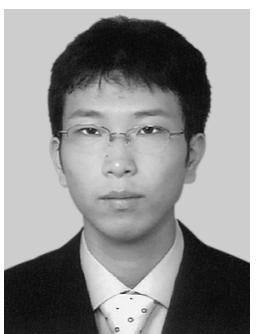

Yoshifumi Kawamura received the B.E. degree in electronic and information engineering from Hokkaido University, Sapporo, Japan, in 2008. He is currently working toward the M.E. degree at the Graduate School of Information Science and Technilogy, Hokkaido University, Sapporo. His research interest is EMC issues. $\mathrm{He}$ is a student member of the IEEE.

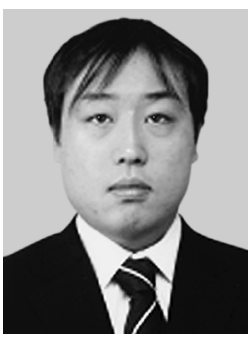

Takashi Hikage received the B.E., M.E. and Ph.D. degrees in electronic and information engineering from Hokkaido University, Sapporo, Japan, in 1997, 1999, 2002, respectively. From 1999 to 2003, he was with the Graduate School of Engineering, Hokkaido University. Now, he is an Instructor of the Graduate School of Information Science and Technilogy, Hokkaido University, Sapporo. His research interests are FDTD analysis and EMC issues. Dr. Hikage is a member of the IEEE.

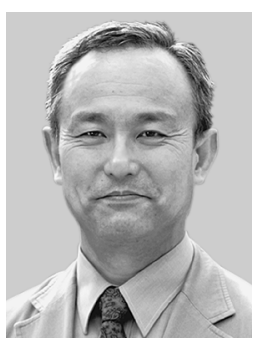

Toshio Nojima received a B.E. in electrical engineering from Saitama University, Japan in 1972 and received M.E. and Ph.D. degrees in electronic engineering from Hokkaido University in 1974 and 1988. From 1974 to 1992 he was with Nippon Telegraph and Telephone (NTT) Communications Laboratories, where he was engaged in the development of highcapacity microwave radio relay systems. From 1992 to 2001 he was with the Research Laboratory of NTT DoCoMo in Yokosuka, where he was Senior Executive Research Engineer and conducted research on radio safety and EMC issues related to mobile radio systems as well as microwave circuit technologies. Since Janualy of 2002 he has been a professor at the Graduate School of Information Science and Technology, Hokkaido University, Sapporo. Dr. Nojima is a member of the IEEE, the Institute of Electrical Engineers of Japan, and the Bioelectromagnetics Society. 